Le CERN fête en cette année

\section{4 ses 60 ans. Cet article}

vise à montrer combien

les vœux des fondateurs

du CERN ont été exaucés.

Le CERN a été un succès

scientifique, un succès pour

la construction d'une Europe

scientifique ouverte sur

le monde, un succès dans

un certain mode d'innovation,

l'innovation ouverte, un succès

dans sa contribution à la paix

dans le monde à travers

la science. Il peut être

considéré avec ses utilisateurs

comme un modèle sociétal

à petite échelle au service

du bien public.

\title{
Le CERN fête ses 60 ans de science pour la paix
}

Michel Spiro (michel.spiro@cea.fr)

Conseiller scientifique au Commissariat à l'énergie atomique et aux énergies alternatives,

(EA Saclay, 91191 Gif-sur-Yvette Cedex

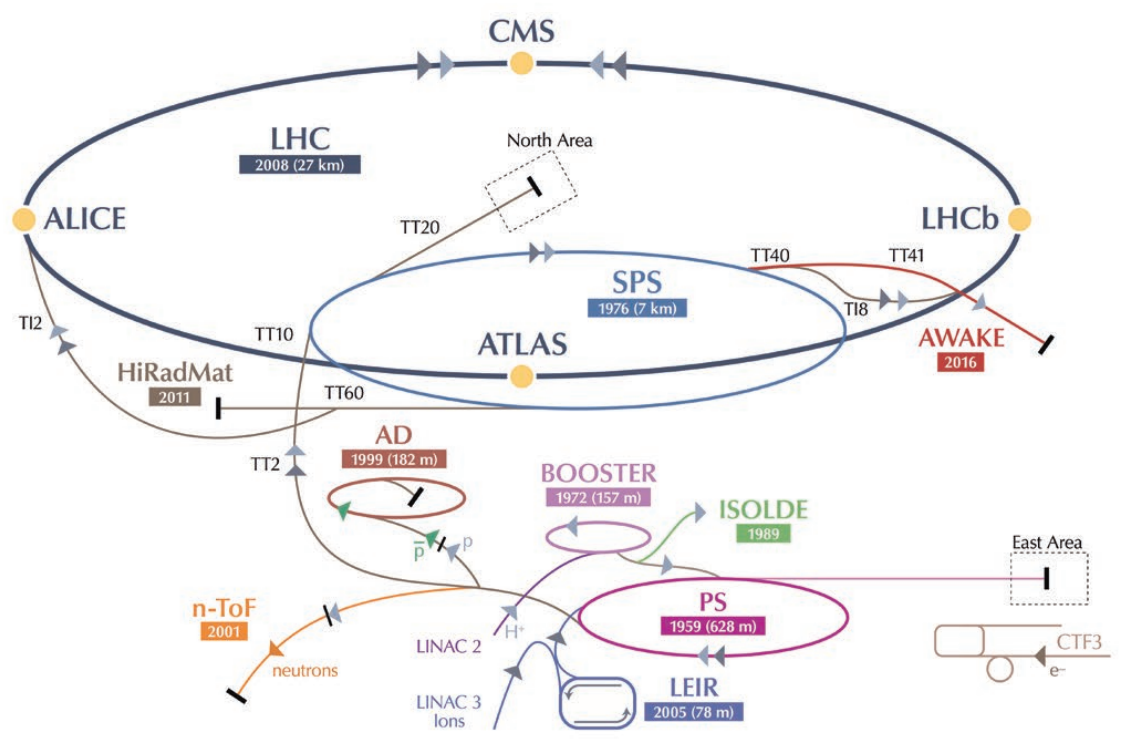

$>\mathrm{p}$ (proton) $\gg$ ion neutrons $\overline{\mathrm{p}}$ (antiproton)

LHC Grand collisionneur de hadrons SPS Super synchrotron à protons PS Synchrotron à protons AD Décélérateur d'antiprotons CTF3 Site d'essai du projet de collisionneur linéaire compact AWAKE Accélération par champ de sillage plasma entraînée par des protons

ISOLDE Séparateur d'isotopes en ligne LEIR Anneau d'ions de basse énergie LINAC Accélérateur linéaire n-ToF Usine à neutrons HiRadMat Test des matériaux sous irradiation

$\sum_{\substack{\frac{m}{5} \\ 0}}^{\infty}$

1. Le complexe des accélérateurs du CERN. Le LHC (ligne épaisse gris foncé) est l’anneau le plus récent dans une chaîne complexe d'accélérateurs de particules. Les machines plus petites sont mises en série pour amener les particules à leurs énergies finales et fournissent des faisceaux à un ensemble d'expériences (voir texte).

Le CERN a été créé par douze États dont la France, peu après la Seconde Guerre mondiale, en 1954, sous l'impulsion de visionnaires qui visaient avec cette organisation à redonner à l'Europe la capacité de rivaliser sur le plan scientifique, en l'occurrence dans la compréhension des constituants de la matière et des lois fondamentales, avec les États-Unis d'une part et l'Union soviétique de l'autre. Parmi ces visionnaires, des Français, citons les scientifiques Louis de Broglie d'abord, puis Pierre Auger et le diplomate François de Rose, mais aussi Francis Perrin, Lev Kowarski et Raoul Dautry. Le CERN, Conseil Européen pour la Recherche Nucléaire, on dirait aujourd'hui
Organisation Européenne pour la Physique des Particules, devait être un exemple de construction européenne et d'ouverture sur le monde, dédié à la recherche fondamentale, et un exemple de recherche et d'innovation collaborative.

Un laboratoire fut créé, qu'on appelle aussi le CERN, pour abriter des accélérateurs de particules qu'aucun des États membres n'aurait pu s'offrir seul. Ce laboratoire, situé près de Genève en Suisse, s'étendit par la suite sur la frontière franco-suisse, si bien que l'on considère que la France et la Suisse en sont aujourd'hui les deux États hôtes. Le CERN a construit ses accélérateurs les uns après les autres comme des poupées gigognes (fig. 1). 


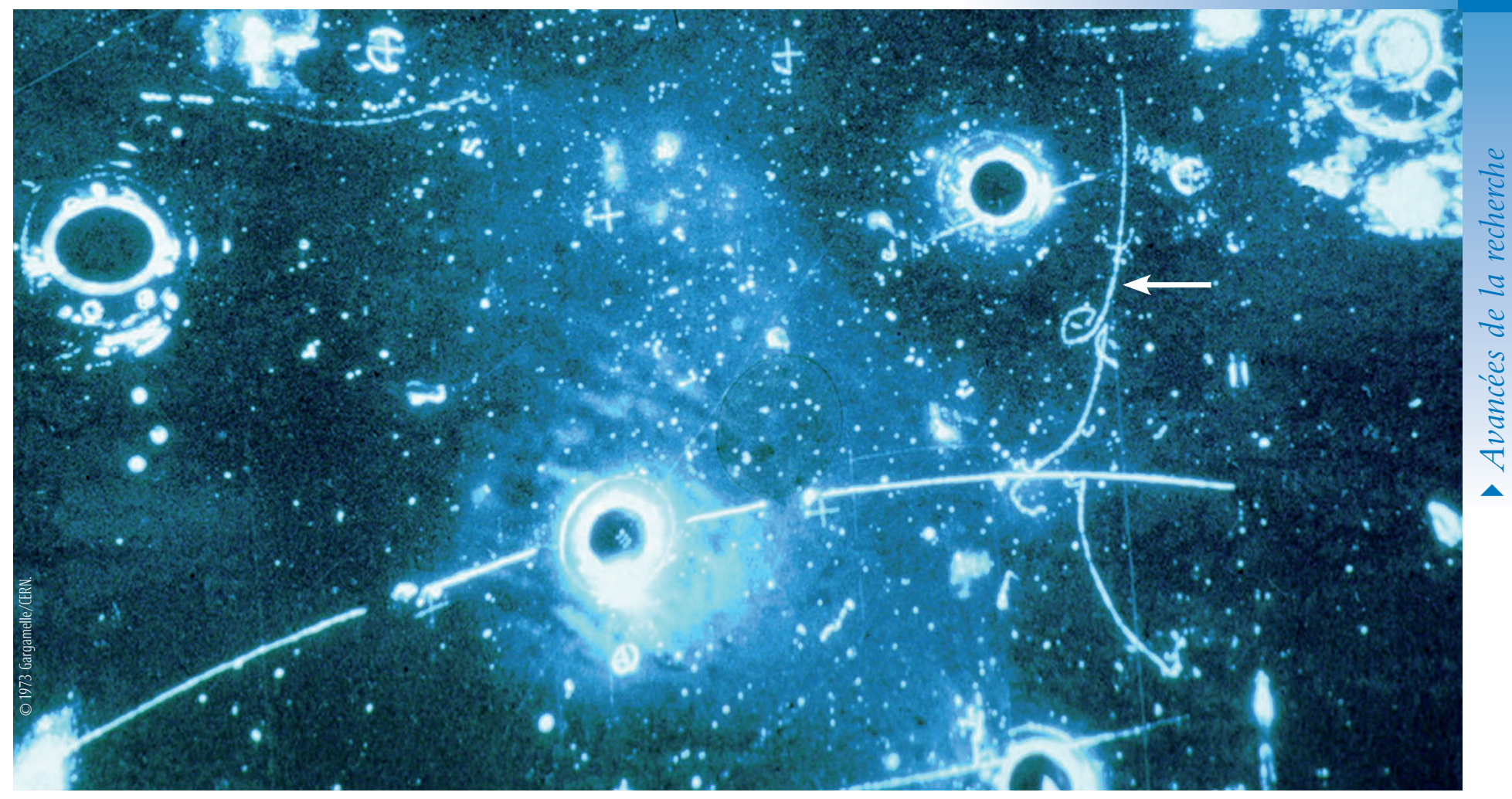

2. Cliché pris dans la chambre à bulles Gargamelle du premier événement observé provenant d’une interaction dite par « courant neutre faible ». Un neutrino muonique incident, invisible et arrivant par le haut du cliché, projette vers le bas un électron (trace verticale, indiquée par une flèche), redonnant un neutrino au lieu de se convertir en muon.

\section{Les premières expériences: le synchrotron à protons PS et la découverte des courants neutres}

Le premier accélérateur, celui qui, dans les années 60, faisait entrer le CERN à la frontière des hautes énergies fut le PS, synchrotron à protons, machine circulaire de 630 mètres de circonférence, qui accélère les protons jusqu'à $26 \mathrm{GeV}$. Un synchrotron synchronise la montée en énergie des particules, réalisée par des cavités accélératrices, avec la montée en champ magnétique dans les aimants de manière à ce que les particules restent dans l'anneau. La limite en énergie des particules est donnée par la taille de l'anneau et le maximum des champs magnétiques que l'on peut atteindre. Au-delà, les particules quitteraient l'anneau vers l'extérieur, n'étant pas suffisamment maintenues sur leur trajectoire. Les principaux acteurs français de l'époque furent Bernard Grégory, directeur adjoint de Victor Weisskopf, premier directeur général du CERN et prix Nobel de physique, Louis Leprince-Ringuet et Francis Perrin, ardents avocats du CERN, et Charles Peyrou qui y construisit les premières chambres à bulles. Les clichés des chambres à bulles (fig. 2) ont été reproduits très largement, depuis les livres scolaires jusqu'aux revues artistiques.
Le laboratoire CERN a acquis ses plus grandes lettres de noblesse autour de découvertes majeures ayant conduit à l'élaboration et à la vérification de la théorie des particules élémentaires d'aujourd'hui, théorie qu'on appelle le "modèle standard». La première très grande découverte du CERN, celle qui a marqué l'histoire du PS, a été faite grâce à la chambre à bulles Gargamelle, construite en France, à Saclay, sous l'impulsion d'André Lagarrigue, d'Orsay. Cette chambre à bulles, qui a fonctionné de 1970 à 1979, était destinée à l'étude des interactions des neutrinos produits à partir des faisceaux de protons du PS frappant une cible. Ces neutrinos interagissaient dans le liquide lourd remplissant la chambre à bulles.

La découverte a été faite en 1973 par une collaboration d'une trentaine de personnes, dirigée par André Lagarrigue. Il s'agit de la découverte d'interactions de neutrinos, sans électrons ni muons dans l'état final (fig. 2). Elle faisait suite à l'émergence des considérations théoriques par Glashow, Weinberg et Salam, donnant naissance à ce qui devait être appelé plus tard le modèle standard. Ces observations s'interprétaient dans le cadre de ce modèle comme la découverte des interactions faibles, par courant neutre, dont le médiateur est le boson intermédiaire $Z^{0}$, neutre électriquement, qui transforme un neutrino initial en un neutrino final. On pense généralement que cette découverte aurait valu le prix Nobel à André Lagarrigue, s'il n'était décédé prématurément.

Le PS fonctionne toujours, sert d'injecteur à des machines construites depuis et situées en aval ; des expériences ainsi que des tests expérimentaux sont toujours conduits auprès de cette machine, qui subit de régulières cures de jouvence.

\section{Les premiers anneaux de collisions proton-proton}

En parallèle, sous l'impulsion encore de Bernard Grégory, devenu directeur général du CERN, le PS alimentait un nouveau type de machine : les anneaux de collision proton-proton dits ISR (Intersecting Storage Rings) préfiguraient ce que serait plus tard, à plus haute énergie, le LHC - le grand collisionneur à protons. Les ISR, situés cette fois sur le territoire français, faisaient collisionner des protons de $28 \mathrm{GeV}$ venant du PS, tournant dans deux anneaux, en sens inverse, et se collisionnant de plein fouet. Les protons naviguant dans un sens percutent ceux naviguant dans l'autre sens, à des endroits bien précis où les deux anneaux fusionnaient. C'était une première et d'un seul coup on avait des collisions qui, par le miracle de la théorie de la relativité, étaient d'une énergie dans le centre de 


\section{>>}

masse équivalente à celle que l'on aurait obtenue si l'on avait accéléré des protons à une énergie de $1500 \mathrm{GeV}$ et les avait fait percuter une cible fixe, comme on le faisait auparavant avec le PS mais avec des protons de $28 \mathrm{GeV}$ seulement.

Les ISR ont permis de révéler que les interactions proton-proton procédaient parfois à travers des collisions dures (très énergétiques) entre un quark d'un proton et un quark de l'autre proton. Les deux quarks étaient éjectés à grand angle par rapport à la ligne de faisceau, donnant naissance à des particules de grande impulsion transverse par rapport au faisceau.

Les ISR ont été démantelés en 1984. Le seul regret que l'on peut avoir est la " petitesse " des détecteurs, même s'ils étaient très innovants, utilisant les récentes chambres de Georges Charpak qui valurent à ce dernier le prix Nobel en 1992. Cette « petitesse » des détecteurs était bien souvent due à la faiblesse de la taille des collaborations. Les ISR auraient pu faire plus de découvertes si la transition vers des détecteurs couvrant tout l'espace de phase et détectant toutes les sortes de particules, opérés par de très grandes collaborations, ce qui allait être la règle pour les machines à venir, avait vu le jour à l'époque des ISR. Ceci montre bien que la "Big Science" est une nécessité et non une commodité.

\section{Produire et manipuler les antiprotons}

Bernard Gregory fut aussi celui qui lança la construction d'une machine plus grande, le SPS (super synchrotron à protons), alimenté par le PS, et qui allait entrer en service en 1976 et accélérer des protons de $26 \mathrm{GeV}$ à $400 \mathrm{GeV}$. Dans un premier temps, les faisceaux allaient frapper des cibles fixes et produire des faisceaux secondaires de types variés : faisceaux de pions, de muons, de neutrinos... Ces faisceaux fonctionnent encore et des équipes y font des expériences ou des tests expérimentaux.

En parallèle à ces recherches, dès le début des années 70, un physicien accélérateur du CERN, Simon Van der Meer, proposait une méthode audacieuse pour accroître les intensités des faisceaux de protons, tout en affinant leur résolution en énergie. À la fin des années 70, Van der Meer démontrait comment sa méthode pouvait aussi s'appliquer avec succès aux faisceaux d'antiprotons. En effet, pour obtenir suffisamment d'antiprotons et en faire un faisceau de
$10^{10}$ particules que l'on puisse accélérer dans le PS puis dans le SPS, encore fallait-il les produire et les mettre en ordre de marche pour constituer un faisceau, c'est-à-dire un ensemble de particules allant toutes dans la même direction et avec la même énergie. Cette prouesse fut réalisée grâce au PS dont les protons, en frappant sur une cible, produisent les antiprotons. Une partie d'entre eux sont captés à une énergie de $3 \mathrm{GeV}$ dans un anneau dédié de grande acceptance. Ils sont ensuite uniformisés en direction et en énergie par les techniques dites de refroidissement (dans le centre de masse du faisceau, la température est abaissée). Le faisceau refroidi est ensuite injecté dans le PS, puis le SPS.

Grâce à ces développements technologiques, c'est avec les antiprotons que le SPS a acquis la gloire et a donné au CERN le rôle de premier rang mondial. En fait, avec les antiprotons et dans la foulée de l'expérience acquise aux ISR, le SPS a fonctionné en mode collisionneur protonantiproton ; il s'est alors appelé le Sp $\bar{p} S$, de 1981 à 1986, les protons tournant dans un sens et les antiprotons dans l'autre sens, chaque faisceau ayant une énergie de $270 \mathrm{GeV}$ par particule. Dès 1982, une découverte majeure était faite par une des deux expériences placées auprès du collisionneur, l'expérience UA2. C'est l'observation cette fois-ci de jets de particules émis à grand angle avec une grande impulsion transverse par rapport au faisceau. C'est, après les ISR, la confirmation que des collisions dures ont lieu entre des sous-structures ponctuelles du proton et de l'antiproton (les quarks et les gluons).

En 1983, la découverte emblématique des bosons intermédiaires $\mathrm{W}$ et $\mathrm{Z}$, à des masses proches de celles attendues dans le cadre du modèle standard, a été le couronnement des développements sur le SPS. Elle valut, dès 1984, le prix Nobel de physique à Carlo Rubbia, initiateur du projet dans son ensemble et porte-parole de l'expérience UA1, et à Simon Van der Meer, grâce auquel le principe du refroidissement stochastique à été découvert et mis au point, puis appliqué aux faisceaux d'antiprotons qui ont pu être accélérés en grand nombre. Cette découverte a été faite par les expériences UA1 et UA2. Ces expériences, surtout UA1, ont marqué un saut dans la taille et la complexité des détecteurs et dans la taille des collaborations (137 signataires pour UA1, j'étais moi-même 1 sur 137).
Avant d'opérer en mode collisionneur, le SPS avait permis des avancées majeures grâce à des faisceaux secondaires de neutrinos et de muons produits par les protons de $450 \mathrm{GeV}$ frappant une cible fixe. Les mesures avaient permis de conforter la chromodynamique quantique, en montrant l'invariance d'échelle due aux "partons " (sous-structures ponctuelles du proton et du neutron, les quarks, qui peuvent être de valence, les trois quarks caractérisant les protons et neutrons, ou appartenir à « la mer " de paires quark-antiquark) et la multiplication des partons de la mer interagissant lorsqu'on observe les protons et neutrons avec des résolutions de plus en plus fines. La compréhension du proton en termes de quarks et de gluons (les partons) entrait dans un nouvel âge, celui des tests et des mesures de précision de la chromodynamique quantique.

Les mesures avaient permis aussi de préciser la masse des bosons intermédiaires $\mathrm{W}$ et $\mathrm{Z}$, médiateurs des interactions électrofaibles des neutrinos et des muons, même si ces $W$ et $Z$ étaient virtuels et donc indétectables directement. Leurs masses étaient estimées à une centaine de $\mathrm{GeV}$, ce qui avait été la motivation principale de la transformation du SPS d'un accélérateur à protons en un collisionneur proton-antiproton ayant suffisamment d'énergie dans le centre de masse pour pouvoir produire les $W$ et $Z$ à l'état réel et les observer avec les détecteurs UA1 et UA2.

Le complexe de fabrication et de refroidissement des antiprotons fonctionne encore aujourd'hui, mais dans une autre optique. Après avoir capturé et refroidi des antiprotons produits par le PS, ceux-ci sont donc décélérés jusqu'à une centaine de $\mathrm{keV}$, puis stockés dans des pièges électromagnétiques. Des positrons (antiélectrons) sont aussi produits (c'est plus facile que les antiprotons, car leur masse est deux mille fois plus petite et les photons gamma se convertissent "volontiers " en paires électron-positron) et sont aussi stockés dans des pièges électromagnétiques. Les deux pièges sont rapprochés (il existe d'autres techniques) et des atomes d'antihydrogène sont ainsi fabriqués, à leur tour piégés, cette fois à l'aide de gradients de champs magnétiques, car les atomes sont neutres mais possèdent des moments dipolaires électrique et magnétique. On arrive ainsi à stocker des milliers d'atomes d'antihydrogène (ce n'est pas beaucoup en termes de grammes, 
mais c'est suffisant pour étudier leurs propriétés) pendant plus de 15 minutes. Les premiers atomes d'antihydrogène avaient été produits au CERN en 1995 par une technique différente. La prochaine étape sera d'étudier la spectroscopie de ces atomes, l'effet de la gravitation sur ces atomes et de comparer tout cela aux atomes d'hydrogène. Tout écart dans ces comparaisons serait révolutionnaire au regard des théories actuelles, mais il faut bien qu'on arrive à comprendre pourquoi l'univers est fait d'hydrogène (de matière en général), et qu'il n'y a pas de trace d'antihydrogène (d'antimatière en général). Les années qui viennent devraient nous en apprendre beaucoup sur ce sujet. Le CERN est largement en pointe sur ces recherches.

\section{Le grand collisionneur électron-positron (LEP)}

Après la découverte des bosons intermédiaires, le CERN allait se lancer dans une entreprise audacieuse : passer des protons aux électrons et donc accélérer des électrons dans ses machines. Le but était de réaliser des collisions électron-positron, d'abord à $90 \mathrm{GeV}$ pour produire de manière très pure des millions de bosons $Z^{0}$, puis dans une deuxième phase monter à $200 \mathrm{GeV}$ pour produire des paires de bosons intermédiaires chargés $\mathrm{W}^{+}$et $\mathrm{W}^{-}$, tout ceci afin d'étudier avec précision le modèle standard, tester ses prédictions et peut-être découvrir le boson de Higgs s'il avait une masse inférieure à environ $100 \mathrm{GeV}$ (le modèle standard au démarrage du LEP ne prédisait pas sa masse, si ce n'est qu'elle devait être inférieure à environ $1000 \mathrm{GeV}$, soit $1 \mathrm{TeV}$ ). Il faut bien réaliser que le collisionneur proton-antiproton ne produisait que des centaines de $Z^{0}$ et des milliers de $\mathrm{W}$, ceci dans un environnement très chargé, la collision quark-antiquark ayant produit le $\mathrm{Z}^{0}$ ou le $\mathrm{W}$ étant entourée de « quarks spectateurs " donnant naissance à un bruit de fond de particules se mélangeant au produit de désintégration des bosons. Plus encore, un $\mathrm{Z}^{0}$ ou un W n'était produit que sur plusieurs milliards de collisions pour lesquelles la collision sous-jacente quark-antiquark n'avait pas les caractéristiques nécessaires pour produire un boson intermédiaire. Au LEP, en ajustant l'énergie de la collision électron-positron pour qu'elle soit égale à la masse du $\mathrm{Z}^{0}$, chaque collision ou presque conduisait à la production du $\mathrm{Z}^{0}$.
Le problème, du point de vue des accélérateurs du CERN, était que lorsque les électrons tournent dans ces machines circulaires, ils rayonnent de la lumière et perdent de l'énergie (les protons rayonnent beaucoup moins parce qu'ils sont 2000 fois plus massifs). Plus le rayon de courbure est petit, plus ils rayonnent. Plus leur énergie est grande et plus ils rayonnent. Il était clair que les $7 \mathrm{~km}$ de circonférence du SPS ne permettaient pas d'accélérer des électrons ou des positrons à $45 \mathrm{GeV}$ et encore moins à $100 \mathrm{GeV}$, énergies requises pour la phase 1 (production de $Z^{0}$ de masse environ $90 \mathrm{GeV}$ ) et la phase 2 (production de paires de $\mathrm{W}^{+}, \mathrm{W}^{-}$). Pour atteindre $200 \mathrm{GeV}$ dans le centre de masse avec une puissance électrique raisonnable (200 MW maximum), il fallait un anneau de $27 \mathrm{~km}$. Cet anneau souterrain fut construit, avec le SPS comme injecteur.

Les premières collisions furent observées le 14 juillet 1989. Dix millions de $Z^{0}$ furent analysés, le modèle standard passa d'une hypothèse vérifiée à $10 \%$ près à une théorie de précision vérifiée au pour mille. En étudiant les désintégrations des $Z^{0}$, le LEP a permis de montrer qu'il y avait trois espèces de neutrinos et pas plus. De plus, il put, entre autres choses aussi, démontrer la liberté asymptotique de la chromodynamique quantique en montrant que la constante de couplage des interactions fortes, leur intensité, diminuait avec l'énergie, laissant entrevoir une convergence des trois constantes de couplage, forte, électromagnétique et faible vers $10^{15} \mathrm{GeV}$, échelle de grande unification, convergence pas exacte mais qui pourrait le devenir grâce à la supersymétrie. La confirmation récente, quoique contestée, d'une échelle cosmologique de $10^{16} \mathrm{GeV}$ correspondant à la période d'inflation exponentielle de l'univers primordial, grâce à la détection d'un fond d'ondes gravitationnelles par BICEP2, conforterait ce scénario. Le LEP montra aussi que même la masse des particules évoluait en fonction de l'énergie, conformément à la théorie de la renormalisation, laissant là encore la possibilité d'unification des masses à l'échelle de grande unification.

Avec sa montée en énergie, à partir de 1996, de $100 \mathrm{GeV}$ à $209 \mathrm{GeV}$, le LEP permit de mettre en évidence des processus, comme la création de paires de W, prédite par le modèle standard et non encore observée, et de déterminer la masse du quark top, le dernier quark manquant du modèle, avant qu'il ne soit découvert aux États-Unis. Les données furent ainsi auscultées avec soin pour découvrir des phénomènes témoignant d'une physique allant au-delà du modèle standard. Rien ne fut découvert, ni particules supersymétriques, ni dimensions cachées, ni non plus... le boson de Higgs qui, lui, était la clef de voûte du modèle standard et le dernier chaînon manquant à l'époque.

Le LEP allait s'arrêter fin 2000 (avec la détermination d'une fourchette pour la masse du boson de Higgs entre 114 et $200 \mathrm{GeV}$ et l'indication d'un possible boson de Higgs à $115 \mathrm{GeV}$ ) pour laisser la place au LHC. L'indication en question ne sera pas confirmée par la suite.

\section{Le grand collisionneur de hadrons (LHC) et la recherche du boson de Higgs}

Conçu au début des années 1980, approuvé en 1994 par le Conseil du CERN, alors présidé par Hubert Curien, le LHC allait réaliser, grâce à des aimants à très haut champ refroidis à l'hélium superfluide, ses premières collisions frontales proton-proton en 2010 à une énergie des collisions de $7 \mathrm{TeV}$, moitié de l'énergie nominale, suite à des premiers essais infructueux en 2008 [1]. Ceci donne une idée de l'échelle de temps de ces machines aujourd'hui. Le lancement de l'étude de la prochaine machine au CERN, qui pourrait avoir $100 \mathrm{~km}$ de circonférence a eu lieu en février de cette année 2014. On peut imaginer à quelle date elle pourrait voir le jour !...

Le but principal du LHC était la recherche du boson de Higgs, clef de voûte et chaînon manquant du modèle standard. Ou on le découvrait et c'était le triomphe de ce modèle et la découverte d'un nouveau type de particule élémentaire (un boson scalaire associé à un potentiel en forme de chapeau mexicain, mécanisme de Brout, Englert et Higgs, donnant des propriétés non triviales au vide quantique, une sorte de viscosité associée à la masse inertielle des particules), ou on ne le trouvait pas (la masse du boson de Higgs est limitée à $1 \mathrm{TeV}$ dans le modèle standard) et c'était tout le modèle standard de la physique des particules qui défaillait. De plus, dans la foulée, on devait découvrir les particules supersymétriques (nécessaires notamment pour stabiliser la masse du Higgs en dessous de $1 \mathrm{TeV}$ et séduisantes pour réaliser la 


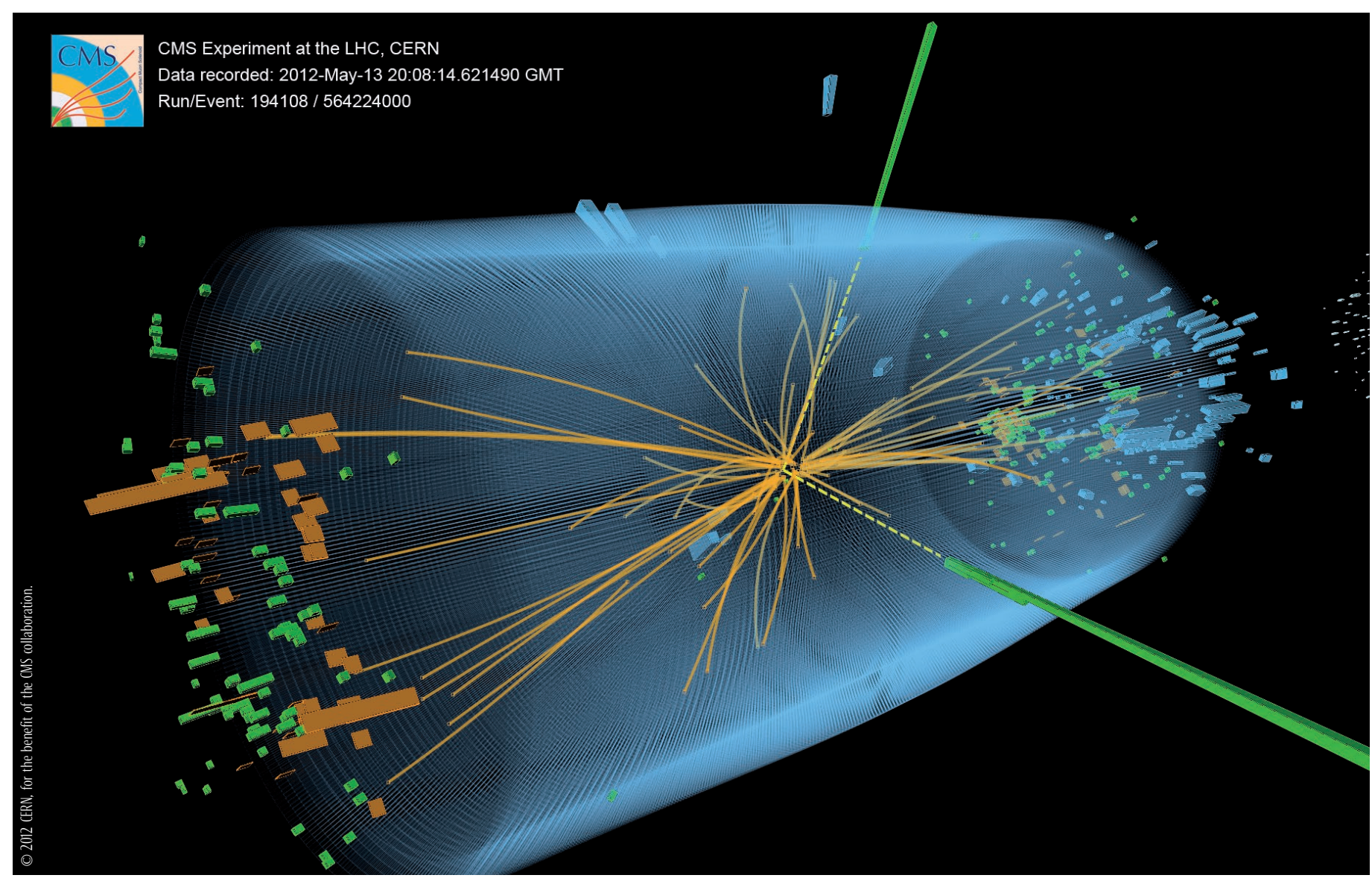

3. Une collision enregistrée en 2012 par le détecteur CMS du LHC, qui a toutes les caractéristiques pour provenir de la désintégration d'un boson de Brout, Englert et Higgs, produisant notamment deux photons (lignes vertes).

\section{$\gg>$}

grande unification à $10^{15} \mathrm{GeV}$, rêve des physiciens, voire même l'unification avec la gravité). Le boson de Higgs a été trouvé en 2012 [2] (fig. 3), François Englert et Peter Higgs eurent le prix Nobel en 2013 [3], Robert Brout étant malheureusement décédé en 2011, mais il n'y a pas jusqu'aujourd'hui de trace de supersymétrie.

Le LHC a aussi réalisé sur l'expérience ALICE des collisions plomb-plomb permettant de découvrir un nouvel état de la matière, prédit par le modèle standard : le plasma de quarks et de gluons, fluide presque parfait [4].

Peut-être de nouvelles découvertes ouvrant une fenêtre au-delà du modèle standard, seront-elles au rendez-vous en 2015 et dans les années suivantes, car l'énergie des collisions au LHC sera portée de $8 \mathrm{TeV}$ à $14 \mathrm{TeV}$. Le LHC tournera jusqu'en 2030 pour sortir des résultats de grande précision et ainsi chercher, directement ou indirectement, des signes d'une physique au-delà du modèle standard.

\section{Le CERN, capitale mondiale de la physique des particules}

Avec ce capital de machines connectées (fig. 1), le CERN est ainsi devenu peu à peu la capitale mondiale de la physique des particules (fig. 4), la frontière des hautes énergies. Le premier vœu des fondateurs visionnaires était exaucé.

Avec 12 États membres à ses débuts, l'organisation en compte maintenant 21 (en bleu sur la figure 4) et s'ouvre aux frontières de l'Europe, notamment avec Israël et peut-être bientôt la Turquie qui est déjà un État associé, nouveau statut qui devrait être offert notamment aux membres du deuxième cercle (en vert sur la figure 4). Dans ce deuxième cercle, on compte des pays qui n'ont pas seulement contribué aux détecteurs et participé aux expériences, mais aussi à la construction de la machine LHC. Ils sont huit, dont les États-Unis, le Japon et la Russie. Par ailleurs, le CERN a des accords de coopération avec une quarantaine de pays (en rouge sur la figure 4) formant le troisième cercle, dont les chercheurs participent aux grandes expériences auprès du LHC. Au total, près de onze mille chercheurs mènent aujourd'hui des expériences au CERN, dont environ $80 \%$ travaillent sur le LHC. Ces chercheurs sont réunis autour de quatre grandes collaborations qui ont construit, en mode autogestionnaire basé sur une constitution, les grands détecteurs ATLAS, CMS, ALICE et LHCb qui ont pu faire ces dernières années des découvertes majeures. En blanc sur la figure 4 figurent les pays qui jusqu'à aujourd'hui n'ont aucun contact avec le CERN.

La construction du LHC et des détecteurs a révélé des défis majeurs qui n'ont pu être surmontés qu'après l'approbation de la machine et des expériences, ce qui constituait un pari audacieux. Ceci a été le cas pour les aimants de la machine refroidis à l'hélium superfluide, une première, pour le traitement des données qui n'a pu être réalisé que par la mise en réseau à grand débit de tous les centres de calcul des laboratoires impliqués, voire des 


\section{Distribution of All CERN Users by Location of Institute on 14 January 2014}

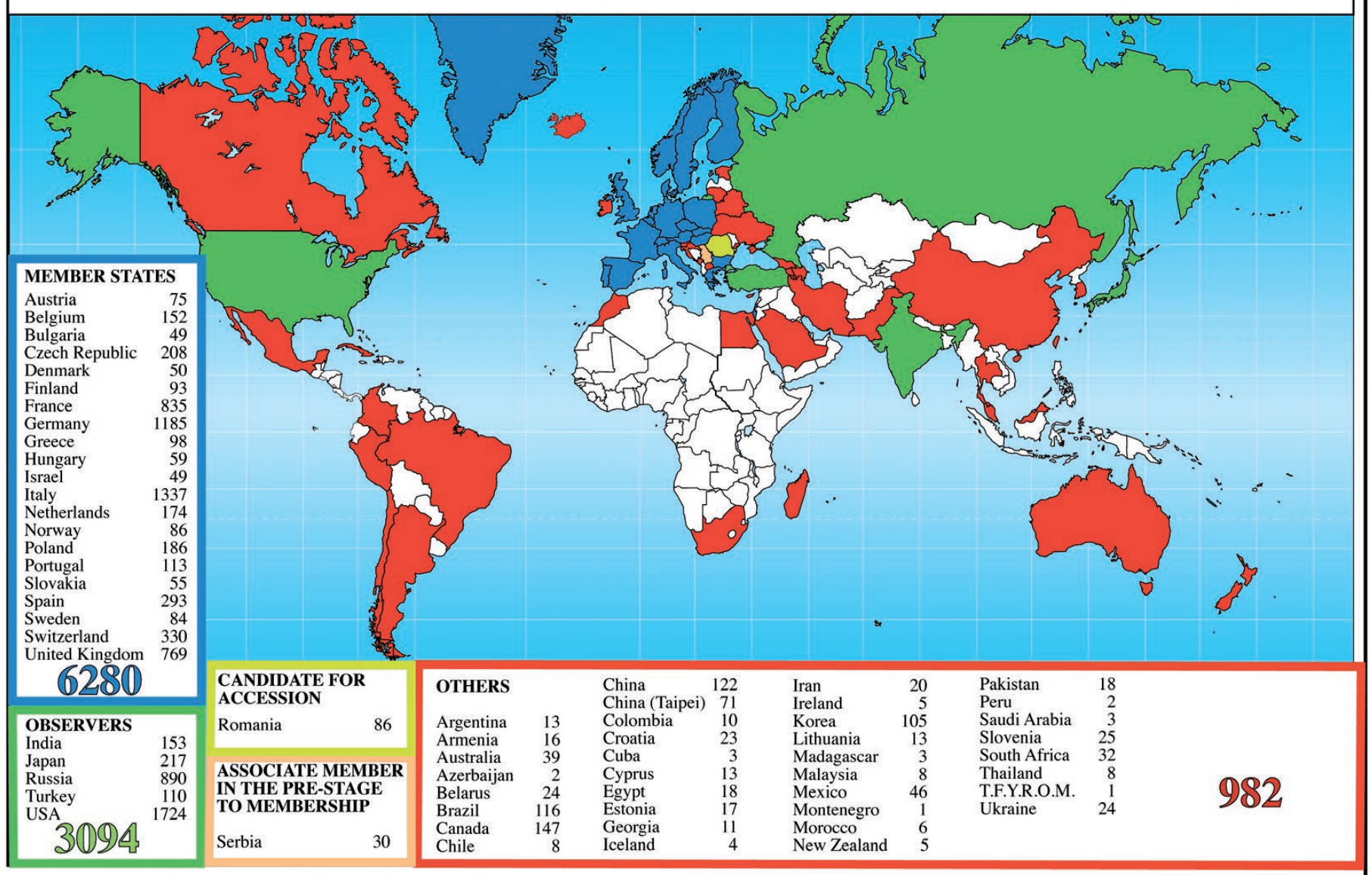

4. La connexion du monde au CERN. Distribution mondiale des utilisateurs du CERN, selon la localisation de leurs instituts, au 14 janvier 2014 (voir texte).

utilisateurs [5] (the World LHC Grid, à l'origine du Cloud computing), pour le contrôle commande, pour les simulations géantes, pour les outils collaboratifs innovants, pour les logiciels complexes... Il est bon de rappeler que c'est déjà au CERN, au démarrage du LEP en 1990, qu'a été inventé et rendu public gratuitement le fameux www qui est devenu le World Wide Web, pour faire échanger les chercheurs du monde entier qui travaillaient déjà à cette époque ensemble sur les expériences du LEP. Ce n'est que grâce à un type original d'organisation, basé sur la collaboration intensive du CERN avec les laboratoires nationaux et universitaires du monde entier, avec la participation d'industriels dès sa conception, que ces défis ont pu être surmontés. Ce modèle qu'on appelle au CERN la " coopétition ", mélange de collaboration et d'émulation, est un modèle ouvert qui pourrait être exportable, mais c'est un autre sujet..

C'est donc, par de multiples critères, la plus grande aventure scientifique humaine. Il a fallu une chaîne de solidarité multi- latérale sans failles et dans la durée entre la communauté scientifique, le soutien de leurs laboratoires, de leurs agences financières et de leurs gouvernements. Les Américains qui avaient un projet similaire, le SSC, n'ont pu tenir la durée et l'ont interrompu de manière unilatérale, par une décision du Congrès en 1993.

La découverte du boson de Higgs résulte de cette stratégie multilatérale, et du savoir-faire qui s'est construit petit à petit pour développer, d'une part de nouveaux outils, permettant de faire travailler ensemble des chercheurs du monde entier (le web en a été l'emblème) et, d'autre part, des méthodes innovantes pour faire collaborer tous ces chercheurs dans une ambiance par ailleurs compétitive (la « coopétition »).

Le LHC modélise une mondialisation réussie ; le deuxième vœu des fondateurs du CERN est lui aussi exaucé : rassembler par-delà les différences de culture, de religion, de citoyenneté, pour une quête commune.

\section{En savoir plus}

J'invite le lecteur à lire l'excellent article qu'avait écrit Maurice Jacob, fameux théoricien français du CERN, dans le Bulletin de la SFP n ${ }^{\circ} 147$ (décembre 2004), pp. 17-20. Le lecteur peut aussi se référer à notre livre Le boson et le chapeau mexicain (Gilles Cohen-Tannoudji et Michel Spiro, Gallimard, Folio Essais, 2013), où ce thème y est beaucoup plus développé. Certains passages de ce texte ont été empruntés à notre livre.

\section{Articles sur le CERN parus dans Reflets de la physique}

1 B. Mansoulié, « Le LHC, grand collisionneur de hadrons", no14 (2009) 5-9.

2• L. di Ciaccio et G. Hamel de Monchenault, «Derniers résultats sur la recherche du boson de Higgs au LHC », n²8 (2012) 15-17 ; « Découverte du boson de Higgs au LHC? », n³1 (2012) 17-19.

3• J. Orloff, « Prix Nobel de Physique 2013 », n³7 (2013-2014) 30-31.

4 P. Crochet, « Collisions d'ions lourds avec ALICE au LHC : les trois premières années ", n³9 (2014) 4-9.

5• F. Malek, « Le calcul scientifique des expériences LHC : une grille de production mondiale », $n^{\circ} \mathbf{2 0}$ (2010) $11-15$ 